\title{
Choroidal Osteoma in a Young Female - a Case Report
}

\author{
Mehr-un-Nisa ${ }^{1}$, Usama lqbal' ${ }^{2}$, Irfan Qayyum Malik ${ }^{3}$, Hira Kanwal ${ }^{4}$ \\ ${ }^{1-4}$ Department of Ophthalmology, Gujranwala Medical College, Gujranwala
}

\begin{abstract}
We present a case of23 year old female, who presented with history of decreased central vision in the right eye for 3 months. Best corrected visual acuity was $6 / 36$ OD and 6/9 OS. Anterior segment was normal OU. Fundus examination revealed a yellowish white peripapillary lesion extending up to the macula in the right eye. A similar lesion was seen in the left eye. OCT macula showed central macular thickness of $193-323 \mu \mathrm{m}$ with cystoid spaces and $290-436 \mu \mathrm{m}$ with serous retinal detachment in the right and left eye respectively. CT scan showed a hyper dense opacity similar to the bony tissue OU. All lab investigations were normal. The patient was diagnosed as a case of bilateral choroidal osteoma. After 6 months no progression or complication was noted.
\end{abstract}

Key Words: Choroid, Osteoma, Choroidal Neoplasm.

How to Cite this Article: Nisa M, Iqbal U, Malik IQ, Kanwal H. Choroidal Osteoma in a Young Female - a Case Report. Pak J Ophthalmol. 2021, 37 (2): 243-246.

Doi: http://doi.org/10.36351/pjo.v37i2.1158

\section{INTRODUCTION}

Choroidal osteoma is a rare benign tumour that arises in the choroid. It can cause visual loss and is found mostly near the optic nerve head. As choroid is the source of nutrition for retina, replacement by bony structure impedes the blood supply of retina leading to retinal ischemia, retinal atrophy and retinal neovascularisation. ${ }^{1}$

It was first described by Van Dyk at Verhoeff society meeting in $1975 .^{2}$ The condition is rarely reported, with the largest case series reporting only 61 patients at a major tertiary center in 26 years. $^{3}$ Choroidal osteoma is unique as it affects otherwise healthy eyes. Most of the affected individuals are reported to be young females in the $2^{\text {nd }}$ or $3^{\text {rd }}$ decade but it can also affect males and patients as young as 2

Correspondence: Usama Iqbal

Department of Ophthalmology

Gujranwala Medical College, Gujranwala

Email: usamaiqqbal@gmail.com

Received: November 3, 2020

Accepted: January 29, 2021 months and as old as 67 years. ${ }^{3}$ Morbidities which can complicate a choroidal osteoma are neovascularization, hemorrhage from the neovessels, accumulation of SRF, degeneration of overlying RPE, calcification and decalcification and vision loss can be due to RPE degeneration, hemorrhage due to CNVs or macular involvement. ${ }^{4,5}$ We report a case of Choroidal osteoma presenting to our department with decreased visual acuity.

\section{Case History}

A 25 Year old lady from a rural area of Gujranwala, Punjab presented in Out-Patient Department of DHQ Teaching Hospital, Gujranwala with complaint of decreased central vision and distorted images in right eye for last three months. There was no significant previous medical and ocular history. Her Visual Acuity was 3/60 in Right eye and 6/9 in Left eye measured on Snellen's visual acuity chart at the time of presentation and metamorphopsia was noted by the patient on Amsler grid. Cycloplegic Retinoscopy was done and best corrected visual acuity was 6/36 in right Eye with -2.00 DS. Anterior segment was normal in both eyes. Fundus examination of the right eye 
revealed a white-yellow peripapillary lesion, extending to macula with sharp round borders and hyperpigmented lesions in the foveal region and periphery. Fundoscopy of the left eye revealed a juxtapapillary lesion extending along vascular arcades sparing the macula but with macular puckering. Fundus photograph is show in (Fig. 1). Disc appeared swollen and to rule out any space occupying lesion in the brain, CT scan was done which was normal for brain but a hyper-dense (resembling bone) opacity was noted in both eyeballs more prominent in the right eye (Fig. 2).

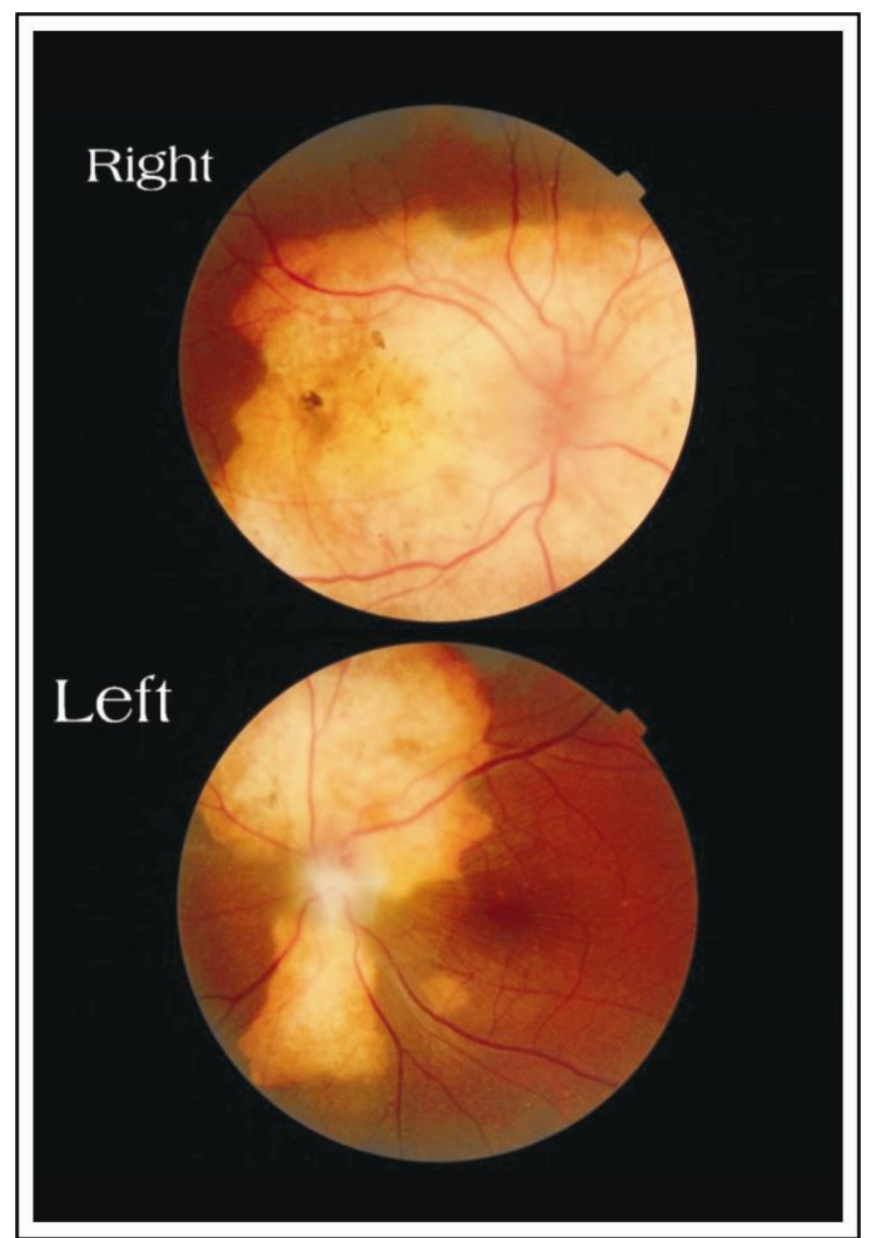

Fig. 1: Fundus photographs.

The patient had no other significant associated ocular symptoms except for mild irritation in both eyes on and off. Family history was insignificant for the related symptoms and no systemic complaint was reported. All the systemic investigations were also normal.

OCT macula of both eyes was done which showed decreased thickness of macula in the centre and increased in the periphery in right eye $(190-323 \mu \mathrm{m})$ with few cystic spaces whereas the left eye showed macular oedema (thickness $290-436 \mu \mathrm{m}$ ) and a serous retinal detachment in extrafoveal region. Visual fields was done on Medmont automated perimeter which showed significant field loss in the right eye whereas central vision was preserved in the left eye which only showed loss of the peripheral field. This can be attributed to the relative macular sparing in the left eye.

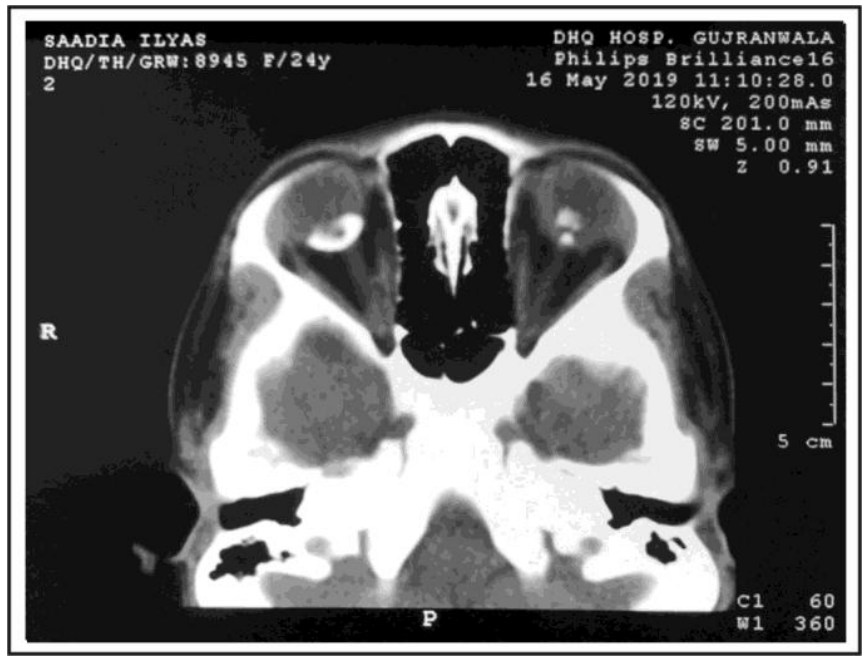

Fig. 2: Axial section-CT scan of orbits.

B-scan right eye showed minimal echoes in the vitreous cavity and a hype reflective shadow just near the optic disc whereas left eye showed hyper reflective echoes near the optic disc and a dark void behind the lesion causing the characteristic pseudo-optic nerve appearance of a choroidal osteoma (Figure 3). FFA showed mottled hyper-fluorescence in the areas of lesion in the choroidal phase and masking of background choroidal vasculature by the bony lesions in both eyes.

After all the investigations and imaging modalities, a diagnosis of bilateral choroidal osteoma was made. The patient was called for a follow up visit after 6 months. No change in signs or symptoms were noted. It was planned to follow up the patient annually to see if any visually debilitating complication develops so that timely management can be done when needed. 


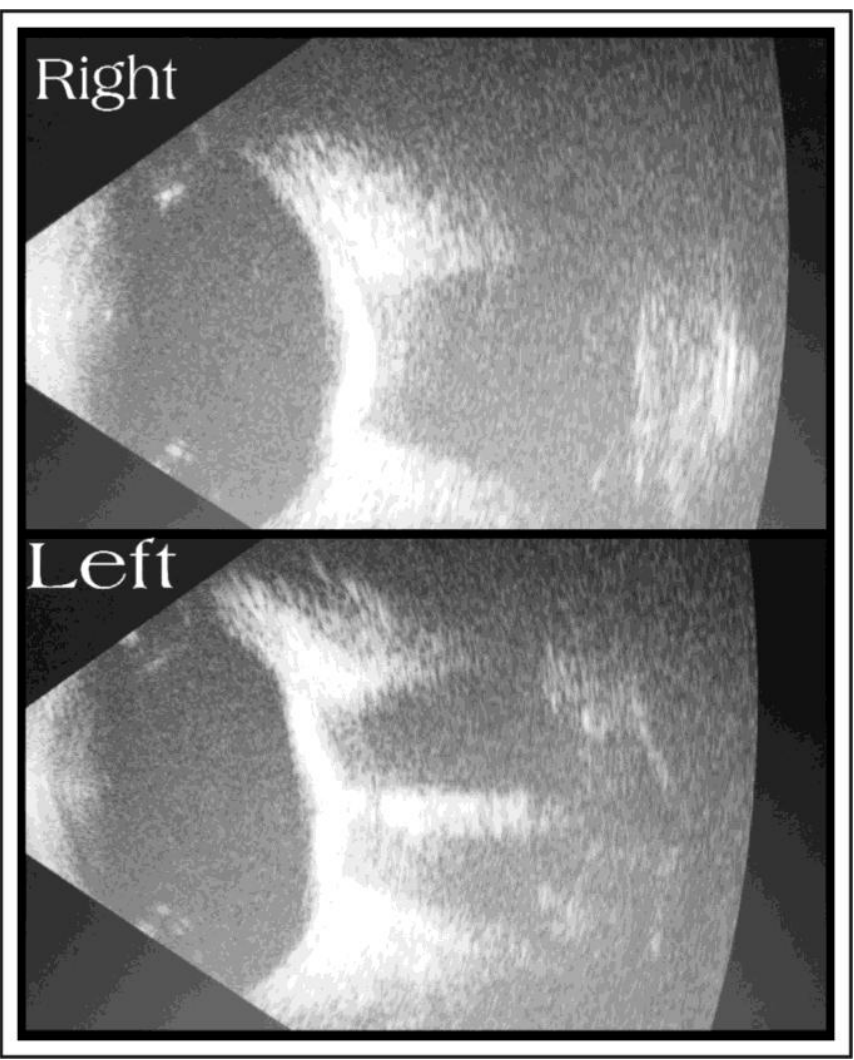

Fig. 3: B scan ultrasound showing choroidal osteoma.

\section{DISCUSSION}

Choroidal osteoma is a rare tumour and cause is not known. It has been postulated that the lesion is a choristoma of the choroid. It can be an incidental finding because many of the patients are asymptomatic. The patients may present with blurry vision, metamorphopsia or scotomas depending on the location of the tumour and associated complications. Vision loss in choroidal osteoma may be gradual or sudden due to Retinal pigment epithelium degeneration, $\mathrm{CNV}$ or macular involvement by the lesion. ${ }^{4,5}$

The case discussed above is even rarer as in $75 \%$ of the patients choroidal osteoma is unilateral. ${ }^{6 .}$ Our patient had bilateral choroidal osteoma although the symptoms and clinical findings were asymmetrical with the right eye being more severely affected than the left eye. Ocular morbidities in choroidal osteoma are due to sensory retinal degeneration and neovascularization, which can be complicated by haemorrhage, CNVM and SRF accumulation. The tumour is slow growing and in the largest cohort, tumour growth was noted in $51 \%$ of the cases whereas decalcification which hinders further growth was noted in $50 \%$ of the total cases at 10 years. ${ }^{3,6}$

Vision loss over 10 years has been reported up to $20 / 200$ or less in $58 \%$ of the cases. ${ }^{7}$ Treatment options for choroidal osteoma are basically concerned with treatment of CNVs and the associated complications. Treatment modalities mentioned in literature include photocoagulation, photodynamic therapy (PDT) and intravitreal anti-vascular endothelial growth factor (VEGF) injection alone or in combination with PDT. ${ }^{8,9,10}$ All these treatment options have been reported to give promising results in terms of visual acuity improvement in case of extrafoveal lesions. There are limited options for degenerated lesions or lesions involving the macular area. Observation is recommended for asymptomatic patients and lesions which are stationary without $\mathrm{CNV}$ s or are decalcified.

It is also important to differentiate this tumour from similar lesions like amelanotic melanoma of the choroid, choroidal metastases, idiopathic sclerachoroidal calcification, choroidal granuloma etc., so that proper management could be done. In this particular case, no CNVs or visual deterioration were observed over a follow up of 6 months. Therefore, no active management was done. The future plan is to observe the patient annually to look for any deterioration of symptoms or growth of CNVs.

\section{CONCLUSION}

Choroidal osteoma can be complicated by vision threatening complications. Proper assessment of the lesion using different imaging modalities and observation is indicated, so that any complication can be picked up and timely management can be done.

\section{Conflict of Interest}

Authors declared no conflict of interest.

\section{REFERENCES}

1. Browning DJ. Choroidal osteoma: Observations from a community setting. Ophthalmology, 2003; 110: 13271334.

2. Gass GD, Guerry RK, Jack RL, Harris G. Choroidal osteoma. Arch Ophthalmol. 1978; 96 (3): 428-435.

3. Shields CL, Sun H, Demirci H, Shields JA. Factors predictive of tumor growth, tumor decalcification, choroidal neovascularization, and visual outcome in 74 eyes with choroidal osteoma. Arch Ophthalmol. 2005; 123: $1658-1666$. 
4. Chen J, Lee L, Gass JD. Choroidal osteoma: Evidence of progression and decalcification over 20 years. Clin Exp Optom. 2006; 89: 90-94.

5. Cherian T, Paulose RM, Reesha KR. Antivascular endothelial growth factor in treatment of choroidal osteoma not associated with choroidal neovascular membrane. Kerala J Ophthalmol. 2017; 29: 237-239.

6. Fine HF, Ferrara DC, Ho IV, Takahashi B, Yannuzzi LA. Bilateral choroidal osteomas with polypoidal choroidal vasculopathy. Retinal Cases Brief Rep. 2008; 2: 15-17.

7. Singh AD, Talbot JF, Rundle PA, Rennie IG. Choroidal neovascularization secondary to choroidal osteoma: Successful treatment with photodynamic therapy. Eye (Lond). 2005; 19: 482-484.

8. Ahmadieh H, Vafi N. Dramatic response of choroidal neovascularization associated with choroidal osteoma to the intravitreal injection of bevacizumab (Avastin) Graefes Arch Clin Exp Ophthalmol. 2007; 245: 17311733.

9. Blaise P, Duchateau E, Comhaire Y, Rakic JM. Improvement of visual acuity after photodynamic therapy for choroidal neovascularization in choroidal osteoma. Acta Ophthalmol Scand. 2005; 83: 515-516.
10. Sharma S, Sribhargava N, Shanmugam MP. Choroidal neovascular membrane associated with choroidal osteoma (CO) treated with trans-pupillary thermo therapy. Indian J Ophthalmol. 2004; 52: 329330 .

\section{Authors' Designation and Contribution}

Mehr-un-Nisa; Postgraduate Resident: Literature search, Manuscript writing.

Usama Iqbal; Postgraduate Resident: Manuscript writing.

Irfan Qayyum Malik; Associate Professor: Supervisor and critical analysis of literature and manuscript.

Hira Kanwal; Investigative \& Ophthalmic Technician: Imaging and visual field analysis, Review. 